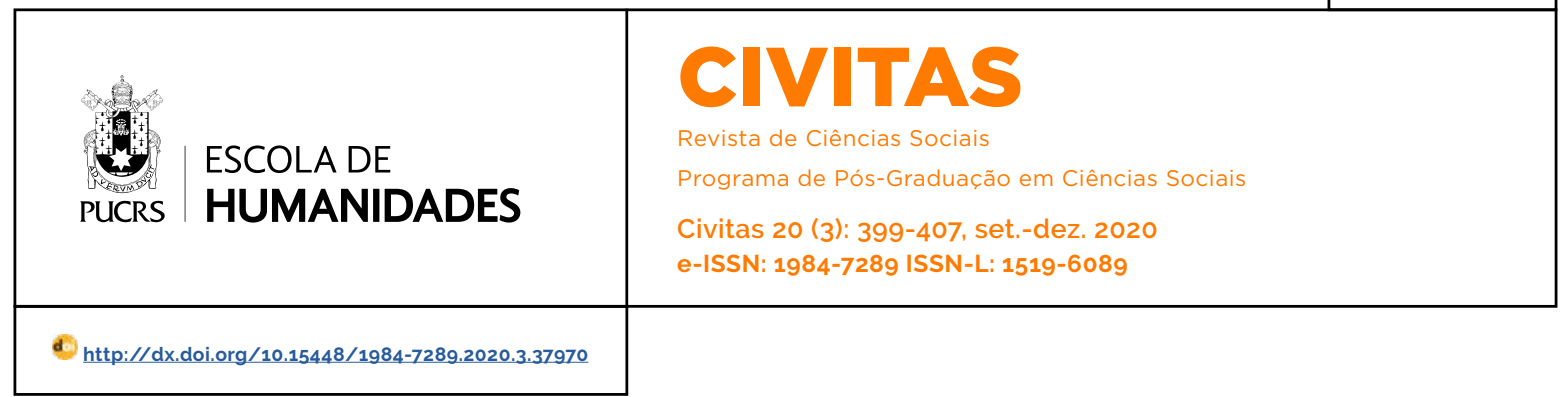

DOSSIÊ: LUTA CONTRA A CORRUPÇÃO: ESTADO DA ARTE E PERSPECTIVAS DE ANÁLISE

\title{
A moralidade da corrupção nas organizações
}

\author{
The morality of corruption in organizations \\ La moralidad de la corrupción en las organizaciones
}

\section{Davide Torsello ${ }^{1}$}

orcid.org/0000-0002-0541-8735

torsellod@ceu.edu

Recebido: 4 maio 2020

Aprovado: 5 maio 2020

Publicado: 23 dez. 2020

\section{(c) (1)}

Artigo está licenciado sob forma de uma licença Creative Commons Atribuição 4.0 Internacional.
Resumo: Quando a corrupção é contextualizada no dia a dia das organizações, o foco ético pode estar no uso da moralidade para transmitir ideias sobre o que é aceitável ou não. A abordagem típica pode ser a de comunicar valores da liderança (o que de fato acontece mais raramente). Uma segunda abordagem pode ser lidar com padrões morais nos padrões diários de socialização dentro e fora da organização. Por fim, o enfoque final diz respeito à problematização das reivindicações morais em contextos sociais em que a corrupção é uma questão cotidiana. Aqui, em vez de delegar a cultura como explicação para a resiliência da corrupção, pode ser proveitoso reunir mais evidências empíricas sobre como os padrões morais são contaminados pelos contextos sociais.

Palavras- chave: Corrupção. Moralidade. Organizações

Abstract: When corruption is contextualized in the daily work of organisations the ethical focus may be on the use of morality to transmit ideas about what is acceptable or not. The typical approach may be that of communicating values from leadership (which indeed happens more rarely). A second approach may be dealing with moral standards in the everyday patterns of socialization within and out of the organisation. Finally, the final focus concerns the problematizing of moral claims in social contexts in which corruption is a daily issue. Here, instead of delegating culture as the explanation for the resiliency of corruption, it may be fruitful to gather more empirical evidence on how moral standards become poisoned in social contexts. Keywords: Corruption. Morality. Organizations

Resumen: Cuando la corrupción se contextualiza en el día a día de las organizaciones, el enfoque ético puede estar en el uso de la moralidad para transmitir ideas sobre lo que es aceptable y lo que no. El enfoque típico puede ser comunicar valores de liderazgo (lo que de hecho ocurre con menos frecuencia). Un segundo enfoque puede consistir en abordar los estándares morales en los patrones diarios de socialización dentro y fuera de la organización. Finalmente, el enfoque final está en problematizar los reclamos morales en contextos sociales donde la corrupción es un problema cotidiano. Aquí, en lugar de delegar la cultura como una explicación de la resiliencia de la corrupción, puede ser útil recopilar más evidencia empírica sobre cómo los estándares morales están contaminados por los contextos sociales.

Palabras clave: Corrupción. Moralidad. Organizaciones

\section{Introdução²}

Anna é gerente intermediária e passou quase toda a sua carreira na divisão de planejamento urbano do escritório municipal de uma cidade na região da Lombardia, no norte da Itália. Ela concordou em ser entrevistada,

Central European University (CEU), Budapest, Hungria.

2 Publicação original: Torsello, Davide. 2019. The morality of corruption in organizations. In Corruption, Social Sciences and the Law. Exploration across the disciplines, organizado por Jane Ellis. London: Routledge. Kindle. Tradução de Fernanda Rios Petrarca, Laboratório de Estudos do Poder e da Política (Lepp), Universidade Federal de Sergipe (UFS), Aracajú, SE, Brasil. 
mas pediu que a entrevista fosse feita separadamente de outros colegas e, por fim, recusou-se a participar de um grupo focal. Um dos motivos de sua recusa foi o fato de ela ter trabalhado mais perto de Luca, outro gerente intermediário que foi indiciado por aceitar suborno de uma empresa local para vencer a licitação sobre a reconstrução do cemitério da cidade. Anna está nervosa no momento da entrevista, ela sabe que poderia ser a única a estar presente fisicamente quando Luca estava negociando com a empresa, apesar de negar ter visto troca de notas. Depois de uma longa conversa preliminar que não lidava expressivamente com a corrupção, Anna começa a se sentir um pouco mais relaxada e desiste de fazer considerações demoradas sobre moralidade. Uma parte dessas considerações lida com a justaposição (op-position/juxta) entre o que ela define como estigma social e moral de trabalho.

Então é dificil dizer, para mim, como para a maioria dos meus colegas, eu acho... Se o cara viesse um dia e me dissesse: "Eu precisava de um carro novo, foi por isso que o comprei", do nada, e isso é um Mercedes, que eu nunca teria condições, devo acreditar que ele comprou com seu salário? Na verdade, não, o fato de que isso aconteceu... quero dizer, ele disse, que ia comprar um carro, pelo menos. Se, digamos, meu colega voltar ao trabalho e não vejo nenhum vestígio de mudanças em seu estilo de vida, por que devo me preocupar? Pelo menos até que alguém me dissesse que ele realmente construiu uma piscina no valor de 15.000 euros em seu jardim. Isso é moralmente irrepreensivel, não posso aceitar. Não porque eu não sabia, mas porque essa é a vergonha que ele trará para a nossa administração. Este é um estigma social que vamos suportar, graças a uma ou duas figuras na prefeitura. Então, nossos cidadãos pensarão que somos corruptos, se alguém pegar o dinheiro para construir uma piscina real ou comprar um carro novo. Não é como se eu decidisse comprar uma piscina inflável para meus filhos, custa 750 euros ou até 1500, ainda é um presente, é aceitável se não afetar o trabalho e a moral social de nossa organização. O fato é que, atualmente, é dificil e, depois de tantos escândalos, manter uma moral de trabalho sólida se o estigma social cair sobre nós de dentro e de fora. (Anna, 47 anos, entrevistada em 20 jun. 2016).

Anna faz uma pausa e depois parece mais aliviada. Afinal, ela sente que não está acusando ninguém entre seus colegas, apenas está oferecendo uma explicação de como as pessoas não conseguem manter padrões morais sólidos e enraizados na sociedade e na organização à qual ela pertence.

Este artigo aborda diferentes aspectos morais da corrupção, introduzindo uma perspectiva etnográfica que se baseia em dados coletados de entrevistas em diferentes contextos organizacionais. A hipótese de trabalho é que a corrupção é percebida como um ato moralmente inaceitável sempre que as diferentes (e muitas vezes conflitantes) camadas do julgamento moral, identificadas pela literatura antropológica sobre corrupção, dificultam a interpretação das consequências individuais e sociais do ato. Isso não se deve ao âmbito de dificil definição do ato moral, ou seja, o que a moralidade significa apenas em um contexto social, mas às contingências do ato nos contextos individual, social e organizacional. A moralidade é construída pelo individuo que percebe o seu alcance e os limites, criando pontos de referência para situações que acontecem em sua vida profissional ou dentro de uma organização.

Todos os casos descritos neste artigo são de individuos que tomam decisões dentro de contextos organizacionais. Se, e até que ponto, os seus julgamentos morais estão imbuídos de normas e valores e, portanto, estão inseridos no contexto social, não é o objetivo principal deste artigo. O principal objetivo é questionar se uma abordagem que considere as posturas morais conflitantes e multicamadas que os individuos adotam ao lidar com a corrupção pode ser usada para explicar a complexidade desse fenômeno. Talvez fosse mais proveitoso considerar a moralidade como "incorporada à ação" ou parte da relação dialética entre escolhas e práticas na vida cotidiana do trabalho.

O artigo começa com uma visão geral de algumas das principais tendências de pesquisa (e não de descobertas) sobre o estudo etnográfico da corrupção, esboçando um mapa de abordagens possiveis e menos possiveis para esse fenômeno, com algumas reflexões sobre os métodos de pesquisa. Na seção final, será dada atenção ao relacionamento entre o domínio no 
qual as reivindicações morais são construidas e transmitidas aos indivíduos que tomam decisões sobre aceitar ou recusar a corrupção nos contextos organizacionais.

\section{Motivações individuais e ordem moral}

Tim é um especialista em anticorrupção. Ele é convidado por prestigiados centros acadêmicos, escolas de negócios, organizações anticorrupção e organizações profissionais para compliance officer, advogados e outras organizações de especialistas e profissionais para apresentar e dar consultoria sobre anticorrupção. Não é difícil avaliar se essas atividades podem ser bastante lucrativas. Mas o que essas organizações querem de Tim é algo mais do que uma conversa bem calibrada e, às vezes, introspectiva sobre anticorrupção. Tim tem algo mais que isso, ele saiu do túnel, ele era um corruptor e agora ele está do outro lado. Os detalhes sobre como isso aconteceu não são extremamente relevantes para este artigo, a corrupção acontece de maneiras surpreendentes similares em todo o mundo e entre os setores público e privado (Torsello 2016). Mais relevante para este artigo é a soma das motivações que o levaram a pagar propinas, várias vezes, como gerente de vendas de uma grande corporação multinacional que opera em vários países da Ásia, África e América do Sul. Ao contar sua história, Tim está absolutamente calmo, ele é acompanhado por seu advogado pessoal, afinal, um profissional que tinha muitas histórias para contar, além do que me interessa.

Depois que comecei a visitar esse país, percebi que os negócios não estavam indo na direção que eu esperava. Comecei a questionar minhas habilidades de venda e isso se tornou particularmente pesado para mim o tempo todo em que voltei ao meu escritório. Os idosos esperavam mais de mim, em um país que, no passado, registrara grandes volumes de vendas, mas não através de nossa empresa. Foi um grande desafio para mim ser enviado a um país como esse para melhorar nosso desempenho e combater a concorrência. No entanto, depois de duas ou três viagens, nada estava dando certo. Não é positivo questionar a si mesmo, principalmente quando outros colegas estavam se saindo muito melhor. Eu me senti diferente e decidi tentar o meu melhor para melhorar isso. Depois de mais uma semana, encontrei alguém que me indicou que uma pessoa em um escritório ministerial pode ter gostado mais de mim. Eu sabia o que isso significava, mas me recusei a atender a essa solicitação, ofendeu meu senso de ética. Mas o tempo passou e ainda assim minhas vendas não foram impressionantes. Eu fiz algo, mas isso não foi suficiente para fazer a diferença, novamente eu estava para trás. (Tim, 53 anos, entrevistado em 21 mai. 2015).

Tim descreveu as situações em que ele aceitou e como ele decidiu pagar um suborno, e outro. Em suas palavras, ele não estava tentando justificar as suas ações, ele estava se enquadrando no papel de um gerente de vendas bem-sucedido, com uma pontuação muito alta e que não estava mais sentindo seu senso de integridade perdido depois de pagar subornos.

Cheguei a um ponto em que não importava o que estava fazendo, mas como estava fazendo. As vendas estavam se moldando magnificamente e era disso que eu precisava. Na minha empresa, pontuei um bônus após o outro e ninguém estava me perguntando se havia algo incomum, eram negócios todos os dias e bem-sucedidos. Além disso, meu senso de certo ou errado não estava em questão, especialmente quando eu estava sendo hospedado de um resort de luxo para outro. Houve um tempo em que me senti onipotente, não havia nada que eu não pudesse fazer ou obter. Quando você está nessa posição, muitas pessoas o rodeiam e, se você conhece muitas pessoas, sempre há alguém para perguntar quando você precisa de algo. Mas então comecei a me sentir deslocado, foi um processo gradual, comecei a sentir falta da minha família, o número de viagens não aumentou, foi a duração das minhas estadias que se tornou cada vez mais longa. Cheguei a um ponto em que me senti estranho com o que estava fazendo e tive que parar. Se você se encontra em uma situação em que não consegue contar a verdade para sua família, há algo errado no que está fazendo. Esse é o conselho que dou àqueles que estão cambaleando ou que estão prestes a aceitar. peguem o telefone e liguem para sua família; talvez você tenha uma força inesperada para trazer a decisão de volta às suas mãos. (Tim, 53 anos, entrevistado em 21 mai. 2015).

O poder evocativo dessas palavras traz de volta a atenção para os domínios nos quais operam considerações e suposições éticas para a 
tomada de decisões. O caso de Tim está longe de ser isolado. Os desafios que ele enfrentou ao vender em um país onde a corrupção era um problema reconhecido são tipicamente os de muitos empresários. Os seus padrões morais eram supostamente altos nos momentos em que ele começou a visitar o país como ele dizia, "de acordo com minha origem social de um país no qual é incomum subornar". No entanto, essa postura moral mudou drasticamente quando sentiu pressão para melhorar as vendas e cumprir as expectativas da empresa. Como em muitos outros contextos organizacionais, as decisões de Tim não tinham sólidos valores de integridade na organização nos quais ancorar e a desconexão entre o que era considerado moralmente bom e como satisfatório no desempenho, como uma moral do trabalho, começou a se expandir.

A narrativa de Tim, no entanto, enfatiza o primeiro ponto, a decisão individual de recorrer ao suborno, pois ele nunca mencionou a pressão da empresa para a qual trabalhava. Afinal, foi sua própria decisão, e ele próprio usufruiu os frutos e pagou as consequências de tal decisão sozinho.

O segundo aspecto - que diz respeito à forma como a moralidade passou a ser definida como influenciadora do processo de tomada de decisão -foi o recurso à narrativa do relacionamento com a familia. Essa narrativa traz de volta o foco do individuo para o nivel social, através do prisma da familia. Se alguém tem que interpretar as dimensões multicamadas nas quais se diz que os atos morais desempenham papéis na decisão de subornar, aqui entra uma segunda desconexão, aquela entre os padrões sociais (familiares) e pessoais. A narração de Tim enfatiza que o momento em que "estar sozinho em um pais desconhecido" começou a se tornar um assunto delicado para ele. Estava em conflito com o momento em que conhecer muitas pessoas e poder convocá-las para qualquer tipo de solicitação era um nivel separado de ordem moral. Aqui, "ajustar-se às expectativas morais locais" era o elemento-chave da desconexão entre a integridade pessoal e social. Essa desconexão é explicada em termos da dissonância cada vez mais evidente entre a integridade pública e privada que Tim estava expressando em seu relacionamento com a organização para a qual trabalhava e a sua família.

A explicação de que a corrupção gera um tipo (falso) de ordem social na qual é moralmente aceitável e se espera ampliar o escopo dos relacionamentos pessoais para encontrar "a pessoa certa na hora certa" foi fornecida por vários trabalhos etnográficos sobre corrupção (Ruud 2000; Turner 2007; Znoj 2007). A questão que permanece sem resposta é: condições de trabalho especificas em uma organização ou no contexto social e de mercado de suas operações criam o espaço para desconectar as diferentes camadas (pessoais, sociais e organizacionais) através das quais a moralidade está intervindo nos processos de tomada de decisão? E se o fazem, como os individuos reagem ao lidar com essas condições quando parecem intransigentes?

Uma resposta em potencial vem da narrativa italiana introduzida no início deste artigo. Anna conta a história de um acordo de corrupção firmado por um de seus colegas. O modo como Anna entende a escolha de operar em termos ilícitos é usando um padrão moral da sociedade em que o "estigma" (em suas palavra) é trazido à toda a organização por meio da decisão de um individuo. A sua bússola moral é o valor do suborno. Uma piscina de jardim de 15.000 euros ou um carro mais caro são subornos que pertencem ao dominio mais alto da moralidade social, enquanto quantidades menores se relacionam aos padrões éticos do trabalho e são, portanto, mais aceitáveis. No entanto, essa narrativa não assume o ponto de vista de julgar a moralidade apenas de acordo com o valor dos subornos. Ele constrói uma estrutura a partir da qual avalia se os dominios social e de trabalho (organizacional) estão misturados ao definir se determinada conduta é boa ou errada. Ofensas morais mais graves são descritas por ela como aquelas em que a ética social e de trabalho estão entrelaçadas, enquanto ofensas menos valiosas (como ela mesma disse: "as pessoas riem de aceitar um suborno digno de uma piscina inflável para crianças") são aquelas em que esses dois dominios permanecem separados. 


\section{Padrões morais e individuais forjados} na organização

Howard é um famoso advogado em seu pais. Sua empresa de auditoria ficou famosa por lidar com a investigação de um sistema de suborno desenvolvido por uma grande corporação multinacional. Howard trabalhou em uma equipe de dezenas de advogados cuja tarefa, em cooperação com duas grandes firmas de auditoria, era reunir o máximo de informações possivel sobre a história desse escândalo.

Howard teve a incômoda tarefa de interrogar os funcionários e gerentes responsáveis da empresa, alguns dos quais acabaram na prisão. A conversa com ele foi extremamente perspicaz, embora não tanto no volume de informações que pudessem ser coletadas, que eram extremamente controladas e filtradas, como seria de esperar. $O$ forte valor dessa narrativa reside na sensibilidade que Howard demonstrou ao descrever as motivações internas e as causas individuais que levaram à deterioração das práticas de negócios da empresa. A maioria dessas motivações estavam, segundo Howard, enquadradas na categoria "fator humano":

Foi uma cena muito ruim ver gerentes seniores indo para a cadeia. Isso nunca aconteceu antes e ninguém sequer pensaria nisso. Os tempos em que os subornos da empresa eram itens dedutiveis de impostos- como as despesas de relações públicas- haviam desaparecido há muito tempo. Ainda poucos nas altas esferas da empresa se esforçaram para comunicar isso adequadamente, para gerentes que lidam com clientes e grandes projetos, particularmente em paises "sensiveis". Parecia que, quando entramos na empresa, esse tempo havia congelado. O tipo de hierarquia que você esperaria de uma corporação desse tipo estava dominando tudo, e isso afetou a maneira como as pessoas trabalhavam, é claro. Se algo estava bem no topo, era isso. Você não precisava questionar, porque não tinha muitas chances de saber os motivos ou simplesmente como as coisas poderiam ter sido feitas de maneira diferente. Não estou argumentando que o topo estava corrompido, ou como foi o caso, estou dizendo que tudo na empresa e em suas bases internacionais foi estabelecido para que os valores não pudessem ser facilmente transmitidos. Quando se trata de gerentes que foram pegos em atos de corrupção, você não diria. A maioria deles eram pessoas com altos perfis morais. Nenhum deles tinha problemas na familia ou trairia cônjuges ou amigos; eram cidadãos respeitosos. As razões pelas quais eles fariam tais coisas não são pessoais, eles não tinham intenções gananciosas. O fator humano é tal, intransponivel em sua natureza. (Howard, 63 anos, entrevistado em 13 abr. 2014).

Essa narrativa aponta as falhas organizacionais que sublinham a desconexão entre o trabalho e os valores sociais. O retrato vivido fornecido por Howard não é apenas outra história de má administração ou de valores organizacionais falsamente direcionados. Esta é a história de uma multinacional poderosa, que por muitos anos não conseguiu (ou se importou) em construir uma cultura de integridade em suas estruturas operacionais. A consideração de Howard sobre o fator humano que é dificil de gerenciar abrange uma série de aspectos que determinaram um gerenciamento inadequado da integridade. Um deles é que estudos organizacionais recentes apontaram como um foco excessivo em uma abordagem de corrupção organizacional, em vez de outra, levou a uma espécie de impasse teórico (Misangyi, Weaver, e Elms 2008). Por um lado, parte dessa literatura concentra-se na corrupção como desvio ou ação criminal. Ao adotar diferentes abordagens disciplinares, ambos consideram o indivíduo um tomador de decisão antiético. Por outro lado, abordagens mais recentes partem do estudo da corrupção como conivência (Ryvkin e Serra 2016; Palmer 2008; Kawata e Papp 2013). Tais abordagens têm a vantagem de deixar espaço para a busca antropológica de uma explicação moral múltipla ou conflituosa, evidentemente desalinhada das abordagens legalista e criminologista. A abordagem da conivência não está aberta apenas à introdução de explicações multicamadas sobre por que a corrupção acontece, ou como e por que ela é racionalizada e, em alguns casos, normalizada (Ashforth e Anand 2003) mas também o papel das organizações e suas estruturas na promoção de formas antiéticas de comportamento. Nesse sentido, há uma mudança de atenção, graças ao crescente interesse da psicologia social (Darley 2005; Yolles 2009), da organização como estrutura, em direção aos aspectos nas operações cotidianas da organização que levam a padrões de 
socialização, como suas características culturais. Algumas abordagens teóricas do comportamento organizacional enfatizaram, por exemplo, o peso dos padrões internos de socialização na determinação de ajustes nas práticas comportamentais antiéticas dos novos funcionários ou no reforço da ética e dos valores organizacionais por meio do fortalecimento do comportamento pró-social do grupo e da construção de identidades. mecanismos. Essas abordagens, que não compartilham o foco antropológico em uma compreensão mais holística do fenômeno, são, no entanto, promissoras, pois ampliam a atenção da abordagem individual estreita versus regras e leis para uma perspectiva mais diferenciada e com vários atores.

No caso descrito por Howard, o aspecto da conivência é proeminente. Se alguém tivesse que traçar a linha do desengajamento dos padrões de integridade pessoal com o modus operandi da empresa, o ponto de encontro dessas duas camadas estaria no conceito de "fator humano". O trabalho empírico sobre corrupção nas organizações empresariais mostrou que, na maioria dos casos, os executivos seniores, quando solicitados a fornecer uma justificativa para a conduta corrupta ocorrida, forneciam uma explicação "maça podre", que poderia confundir com a explicação do fator humano. No entanto, o "fator humano" é algo mais: ele contrasta o nivel social, no qual os padrões de moralidade dos gerentes corruptos são construídos, com os aspectos funcionais e estruturais da organização que criam (se isso realmente acontece ou não) um ambiente de trabalho adequado para comportamentos antiéticos. O fator humano é o oxímoro através do qual as falhas organizacionais são reduzidas ao nivel da moralidade social e servem à função de ofuscar quem é o culpado. Pouco importa se o gerente subornou para garantir negócios ou seu superior que instigou uma cultura de urgência e desempenho. O que importa é que o fator humano possa fornecer um escudo à empresa ou ao individuo, adotando posturas morais substituiveis e intercambiáveis que possam trabalhar juntas, mas que não se encontram em nenhum momento.

\section{Motivações organizacionais}

Estudos sobre corrupção nas organizações têm apontado cada vez mais a importância de prestar a devida consideração às condições organizacionais sob as quais o ato corrupto ocorre (Luo 2005; Misangyi, Weaver, e Elms 2008). Isso implica, como mencionado acima, na adoção de uma abordagem que considere a corrupção como "conivência" e não como um mero ato criminoso com sua valência individual. Nessas abordagens, há uma tendência a observar alguns dos aspectos que caracterizam a cultura das organizações, como liderança, comunicação, motivação, clareza de tarefas, mudança organizacional (incluindo aquisições e fusões) e criação e transmissão de valor. No entanto, além de alguns artigos que fornecem revisões de literatura, essas contribuições são dispersas e não há clareza suficiente sobre quais fatores e aspectos da cultura das organizações são mais importantes para determinar como os padrões de conivência encontram forma.

Nos casos descritos acima, os funcionários entrevistados apontaram três aspectos principais da cultura de sua organização manchados pela corrupção. O primeiro é liderança e comunicação. Durante o grupo focal que se seguiu às sessões de entrevista, um gerente de nivel intermediário indicou que:
É claro
que o primeiro passo, quando essas coisas acontecerem, seria culpar o topo da gerência. Não tenho vontade de fazer isso, não estou em posição de julgar, mas o que importa é que haja clareza de informações, ordens e tarefas... em algum momento eu, tivemos a sensação de que a comunicação ficou presa em algum lugar, houve um cortina escura que não conseguimos penetrar, e isso é arriscado. (Giulio, 44 anos, entrevistado em 12 maio 2016).

Da mesma forma, outro participante do grupo focal acrescentou:

Isto é certo. Se eu tenho em todos os momentos clareza do que meu chefe espera isso de mim, e é claro que a ética é um padrão importante para o nosso trabalho, tenho idéias claras. Mas se tivermos que continuar comprometendo (navigare a vista) a maior parte do tempo, e é isso que costumamos fazer, quão claro posso ser o 
que é esperado de mim e como isso mudou? (Martina, 34 anos, entrevistada em 12 mai. 2016).

Ambas as manifestações de cautela sobre os padrões pouco claros de comunicação da liderança evocam uma cultura organizacional na qual não apenas os canais de comunicação com a liderança dificilmente são acessiveis pelos niveis mais baixos, mas também é possivel criar ambiguidade quando essa comunicação deficiente é misturada com condições duradouras de impermanência e mudança. Essas últimas descobertas surgiram também em outros estudos etnográficos mundiais de organizações sob ameaça de corrupção (Torsello 2016).

Um segundo aspecto que diz respeito aos elementos culturais organizacionais é a transmissão e sustentabilidade de valores. A moralidade da integridade no trabalho dos administradores públicos é frequentemente ameaçada por casos que a tornam instável e confuso. Uma entrevistada contou a seguinte história:

\begin{abstract}
A introdução do especialista em anticorrupção como parte da nova legislação anticorrupção é um desenvolvimento recente contestado na cultura das administrações públicas. Poucos de nós entendem o que significa estar empregando e pagando uma pessoa extra que deve ser especializada em anti-suborno. O fato é que, às vezes e eu não estou falando apenas do meu local de trabalho, essa pessoa é uma autoridade já existente na organização, movida para essa ocupação. Não há nada errado se ele é uma pessoa tutta d'un pezzo (caracterizada por forte integridade pessoal), no entanto, esse nem sempre é o caso. Se esse especialista tem em sua carreira anterior algum esqueleto no armário, como você acha que devemos nos sentir com toda essa nova legislação complexa e onerosa? (Paola, 37 anos, entrevistada em 12 maio 2016).
\end{abstract}

A integridade e a integridade situacional são vistas na literatura como aspectos determinantes que cimentam ou quebram os padrões éticos de uma cultura organizacional. O ponto de virada difícil é quando se aceita a existência de condições de integridade situacional: isso acontece quando o padrão moral que se difunde em uma organização é aquele que pode tolerar a corrupção ao perseguir os objetivos e metas da organização. O duplo padrão moral torna-se aquele que encontra aceitação e, eventualmente, pode se tornar uma boa solução se levar a uma solução positiva de problemas ou a atingir as metas, mesmo que exija comprometer os padrões éticos e morais. A corrupção é aceita, talvez não de bom grado, mas sob condições ditadas por prazos (principalmente para contextos de administração pública) ou pela necessidade de atingir metas (em organizações empresariais). Seria um campo para uma investigação mais aprofundada se os gerentes que adotam vários padrões éticos na tomada de decisões sobre corrupção são pessoas geralmente estimadas e respeitadas dentro da organização, uma vez que são vistas como fazendo o melhor para isso, pelo menos até que escândalos surjam. O mesmo caso deste artigo é testemunho desse aspecto. Os colegas daqueles que aceitaram suborno não indicaram a existência de práticas difundidas e repetidas de conduta imprópria; eles foram descritos principalmente em termos de pessoas "attaccate al lavoro" (dedicado ao trabalho) ou mesmo se preocupando com o bom desempenho e serviço prestado pela a própria organização.

Finalmente, outra área que se mostraria promissora para pesquisa é aquela que investiga a relação entre integridade situacional e padrões de sociabilidade nas organizações. É, em essência, relativamente raro encontrar casos de personalidades distorcidas ou anti-sociais marcando os caracteres de individuos que aceitaram ou pagaram subornos. Isso sugere que os padrões morais que analisam a sociabilidade e a importância dos padrões de socialização nas organizações podem ser realmente agentes que geram, facilitam ou pelo menos toleram a corrupção. ${ }^{3}$ Essa abordagem reforçaria descobertas mais gerais segundo as quais, em alguns contextos sociais, pequenos niveis de corrupção são tolerados como padrões de sociabilidade ou contrapartida da burocracia racional (e transparente) fria e anônima, presente em diferentes perspectivas nas disciplinas das 
ciências sociais (Torsello 2015).

\section{A moralidade importa mesmo?}

A visão rápida da vida organizacional oferecida pelos casos considerados neste artigo fornece uma imagem complexa e contraditória em termos das implicações morais do trabalho em condições de integridade instável. Os padrões morais são, sem dúvida, contrastados com o conteúdo e o significado das práticas cotidianas. Uma explicação amoral ao estilo do famoso argumento de Banfield no sul da Itália (Banfield 1958) não captura a complexidade da situação. A corrupção não ocorre condicionalmente à existência de uma explicação circular do tipo "todo mundo faz isso, eu também". Também não se pode presumir facilmente, como algumas publicações tradicionais, que a imoralidade ou a falta de moralidade dominam a cena, misturadas talvez com desconfiança generalizada (Uslaner 2008). Se esse fosse o caso, os gerentes que operam sob condições de integridade situacional seriam objetos de desconfiança generalizada a priori, o que por sua vez geraria atitudes cínicas e uma recusa em se envolver em corrupção. A integridade situacional é vista positivamente por alguns membros da organização, que vêem no espirito de serviço a necessidade de comprometer quando metas, objetivos e até empregos estão em jogo.

$O$ argumento de que, sob condições socioculturais externas de fraca moral, as organizações se tornam entidades corruptas, poderia ser adotado aqui, mas isso não esclareceria por que a corrupção surge ou é um fenômeno resiliente. Pelo contrário, a estigmatização da corrupção como prática cultural, como apontou alguma literatura antropológica e recentemente economicista (Smith 2007; Banuri e Eckel 2012), serve para contextualizar e relativizar a moralidade, exaltando sua variabilidade nas sociedades. O relativismo da suposição é que a moralidade não é especifica da cultura, mas, eventualmente, a corrupção é uma questão de cultura, pois é determinada pelas caracteristicas essenciais de sua cultura e, eventualmente, se torna uma característica em si. Essa abordagem não leva em consideração algumas das recentes descobertas acima mencionadas sobre integridade nas organizações, que apontam por que e como os padrões de corrupção são extremamente semelhantes em diferentes organizações, pois colidem com aspectos culturais organizacionais semelhantes, como os descritos na seção anterior. Por outro lado, a abordagem da corrupção como cultura esconde uma realidade importante, o reconhecimento comum de que a corrupção é ruim para todos, também (e especialmente) para os cidadãos dos paises em que é um grande problema. Ao relativizar a corrupção como cultura e a moralidade como axioma contextual da cultura, os aspectos positivos versus negativos desse fenômeno podem eventualmente ser colocados na vanguarda da análise, fornecendo uma versão distorcida da realidade social.

Qual é o uso de explicações morais (moralística) no estudo da corrupção? Como apontado acima, se pontos de vista morais são tomados como parâmetros para medir o impacto da corrupção na sociedade, eles podem ser de pouca ajuda, não apenas para abordar a questão de por que a corrupção acontece, mas, eventualmente, também sobre por que ela é tolerada e aceita em contextos sociais. No entanto, os padrões éticos são aqueles contra os quais os membros de uma organização (onde a corrupção geralmente ocorre) medem seu comportamento no curso das operações diárias de trabalho, em particular no caso de transações comerciais. Nesse caso, remover completamente a moralidade pode ter o efeito contrário de criar um vazio comportamental, um espaço vazio no qual padrões ilícitos de comportamento ocorrem desconectados um do outro e todos como expressões individuais de ganância. A experiência e a pesquisa empírica mostraram abundantemente que esse não é o caso. Na organização em que os funcionários são socializados e aprendem a subornar ou a evitar subornos, mal pode haver casos de crimes individuais. Nessa perspectiva, os padrões morais são importantes, pois são modelos de comportamento, modelados ou não nas características da cultura da organização, mas ainda constituindo maneiras pelas quais as histórias, mitos, casos e exemplos são fofocados, contados ou explicados no melhor das situações. Sob essas premissas, os 
padrões morais são importantes quando a unidade de estudo não é uma sociedade, mas uma organização, como expressão de uma entidade mais ou menos fragmentada que compartilha um estilo de trabalho comum, funcionamento de processos, rituais e relações que compõem sua cultura. A moral é importante, pois regula os padrões transacionais dentro e fora das organizações. Essas transações são avaliadas como éticas ou antiéticas, ou uma mistura das duas, como as abordagens de integridade situacional mostraram.

A descoberta mais significativa deste artigo é que as preocupações morais são relevantes para o estudo da corrupção em dois casos. A primeira é quando a corrupção é contextualizada no trabalho diário das organizações que fazem uso da moralidade para transmitir idéias sobre o que é aceitável ou não. Eles normalmente fazem isso não apenas comunicando valores da liderança (o que realmente acontece mais raramente) mas, mais importante, liderando normas morais nos padrões cotidianos de socialização dentro e fora da organização. Finalmente, o segundo caso diz respeito à problematização de reivindicações morais em contextos sociais em que a corrupção é uma questão cotidiana. Aqui, ao invés de delegar a cultura como explicação para a resiliência da corrupção, pode ser proveitoso reunir mais evidências empíricas sobre como os padrões morais são contaminados pelos contextos sociais. Isso pode ser alcançado levando em consideração o papel dos sistemas juridicos e do pluralismo, condições históricas, transformação institucional e dependência de interesses empresariais amplos e globais.

\section{Referências}

Ashforth, Blake E. e Vikas Anand. 2003. The normalization of corruption in organizations. Research in Organizational Behavior (25): 1-52. https://doi.org/10.1016/ S0191-3085(03)25001-2.

Banfiel, Edward C. 1958. The moral basis of a backward society. Glencoe: Free Press.

Banuri, Sheheryar e Catherine Eckel. 2012. Experiments in culture and corruption: a review. World Bank Policy Research Working Paper 6064. https://doi. org/10.1596/1813-9450-6064.

Darley, John M. 2005. The cognitive and social psychology of the contagious organizational corruption. Brooklyn Law Review 70 (4): 1117-1194.
Kawata, Junici e Melinda Papp. 2013. The meta-language of politics, culture and integrity in Japan. Human Affairs 23 (2): 246-254. https://doi.org/10.2478/s13374-013-0122-8.

Luo, Yadong. 2005. An organizational perspective of corruption. Management and Organization Review 1 (1): 119154. https://doi.org/10.1111/j.1740-8784.2004.00006.x

Misangyi, Vilmos F., Garry R. Weaver e Heather Elms. 2008. Ending corruption: the interplay among institutional logics, resources and institutional entrepreneurs. Academy of Management Review 33 (3): 750-70. https:// doi.org/10.5465/amr.2008.32465769.

Palmer, Donald. 2008. Extending the process model of collective corruption. Research in Organizational Behavior 28: 107-135. https://doi.org/10.1016/j.riob.2008.04.005.

Ruud, Arild E. 2000. Corruption as everyday practice. The public-private divide in local Indian society. Forum for Development Studies 2: 271-94. https://doi.org/10.1 080/08039410.2000.9666142.

Ryvkin, Dmitry e Danila Serra, 2016. The industrial organization of corruption: monopoly, competition and collusion. Working Papers. 10 (1). Department of Economics, Florida State University. https://econpapers. repec.org/paper/fsuwpaper/wp2016_5f10_5fo1.htm.

Smith, Daniel J. 2007. A culture of corruption: everyday deception and popular discontent in Nigeria. Princeton: Princeton University Press.

Torsello, David. 2015. Corruption as social exchange; the view from anthropology. In Debates of corruption and integrity, organizado por Peter Hardi, Paul M. Heywood e Davide Torsello, 159-183. New York: Palgrave.

Torsello, Davide, ed. 2016. Corruption in the public administration: an ethnographic approach. Edward Elgar: Cheltenham.

Turner, Simon. 2007. Corruption narratives and the power of concealment: the case of Burundi's civil war. In Corruption and the secret of law: a legal anthropological perspective, organizado por Monique Nuijtel e Gerhard Anders, 125-142. Aldershot: Ashgate.

Uslaner, Eric. M. 2008. Corruption, inequality and the rule of law. Cambridge: CUP

Yolles, Maurice. 2009. A social psychological basis of corruption and sociopathology. Journal of Organizational Change Management 22 (6): 691-731. https://doi. org/10.1108/09534810910997078.

Znoj. Heinspeter. 2007. Deep corruption in Indonesia: discourses, practices, histories. In Corruption and the secret of law: a legal anthropological perspective, organizado por Monique Nuijtel e Gerhard Anders 53-76. Aldershot: Ashgate.

\section{Davide Torsello}

Antropólogo do Central European University em Viena, Áustria no departamento de Economia e Negócios. Diretor do CEU Center for Integrity in Business and Government. 